\title{
STEADY STATES AND DYNAMICS OF 2-D NEMATIC POLYMERS DRIVEN BY AN IMPOSED WEAK SHEAR*
}

\author{
HONG ZHOU ${ }^{\dagger}$ AND HONGYUN WANG ${ }^{\ddagger}$
}

\begin{abstract}
We study the 2-D Smoluchowski equation governing the evolution of orientational distribution of rodlike molecules under an imposed weak shear. We first recover the well-known isotropic-to-nematic phase transition result [G. Marrucci and P. L. Maffettone, Description of the liquid-crystalline phase of rodlike polymers at high shear rates, Macromolecules, 22, 4446-4451, 1989]: in the absence of flow the isotropic-nematic phase transition occurs at $U=2$ where $U$ is the normalized polymer concentration, representing the intensity of the Maier-Saupe interaction potential. Then we show that in the presence of an imposed weak shear there is a threshold $\left(U_{0} \approx 2.41144646\right)$ for $U$ : When $U<U_{0}$, steady state solution exists; otherwise there is no steady state. Furthermore, we carry out multi-scale asymptotic anlaysis to study the slow time evolution driven by the weak shear. It is revealed that, to the leading order, the order parameter of the orientational distribution is invariant with respect to time whereas the angular velocity of the director is position-dependent. When $U<U_{0}$, the director of the orientational distribution converges to a stable steady state position; when $U>U_{0}$, the angular velocity of the director is always positive and the orientational distribution will not reach a steady state. Finally, the effect of weak shear on the phase diagram is investigated. It is found that the phase relation under weak shear can be obtained from the pure nematic phase relation through a simple algebraic transformation.
\end{abstract}

Key words. Nematic polymers, Kinetic theory, Maier-Saupe potential, Shear flow, Perturbation analysis

AMS subject classifications. $35 \mathrm{Kxx}, 70 \mathrm{Kxx}, 82 \mathrm{D} 60$

\section{Introduction}

The rheological properties of nematic liquid crystals are strongly affected by the dynamic behavior of the molecular alignment $[1,20]$. The standard Doi-Hess kinetic theory treats the nematogenic molecules as rigid rods, and describes the ensemble with an orientational distribution function. The dynamics of the orientational distribution function is governed by the nonlinear Fokker-Planck (Smoluchowski) equation. The steric mutual interaction of rodlike molecules is modeled by the Maier-Saupe interaction potential $[26,27,4]$. The complex behavior of 3-D nematic polymers subjected to an imposed shear flow has been investigated numerically by solving the Smoluchowski equation using spherical harmonic expansions [13, 14, 15, 16, 17, 18, 19, 22]. However, theoretical analysis on this subject is still at its early stage. Recently the mathematical society started to investigate the Smoluchowski equation theoretically $[5,6,7,8,10,11,12,24,25,29,30,31,32]$. The purpose of this work is to extend the analytical studies to Smoluchowski equation under an imposed weak shear.

The paper is organized as follows. Section 2 is a brief review of the results for pure nematic polymers. In section 3 we study steady states of nematic polymers in the presence of an imposed weak shear. Section 4 covers the slow time evolution of orientational distribution using the approach of multi-scale asymptotic analysis. In section 5 we investigate the behavior of the Smoluchowski equation near the isotropicnematic phase transition $(U=2)$. The main results of the paper are summarized in section 6 .

\footnotetext{
${ }^{*}$ Received: October 26, 2006; accepted (in revised version): November 27, 2006. Communicated by Pingwen Zhang.

${ }^{\dagger}$ Department of Applied Mathematics, Naval Postgraduate School, Monterey, CA 93943 (hzhou@ nps.edu)

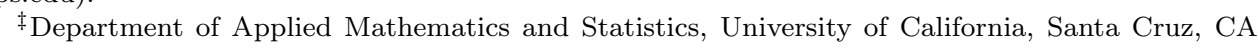
95064 (hongwang@ams.ucsc.edu).
} 


\section{Pure nematic polymers}

For polymer rods in the two-dimensional (2-D) space, the orientation of a polymer rod is described by an angle $\theta$ and the orientation direction is mathematically represented by the unit vector: $\mathbf{u}=(\cos \theta, \sin \theta)$. The Maier-Saupe interaction potential is given by $[26]$ :

$$
V_{M S}(\theta)=-k_{B} T U[\langle\cos 2 \theta\rangle \cos 2 \theta+\langle\sin 2 \theta\rangle \sin 2 \theta],
$$

where $U$ is the normalized polymer concentration, representing the strength of the Maier-Saupe interaction potential, and $k_{B} T$ is the Boltzmann constant times the absolute temperature. For mathematical convenience, in this study we set $k_{B} T=1$ (or equivalently, all potentials are normalized by $k_{B} T$ ). In the Maier-Saupe interaction potential (2.1), $\langle\cos 2 \theta\rangle$ denotes the average of $\cos 2 \theta$ with respect to the current orientational distribution of polymer rods $f(\theta, t)$. The time evolution of $f(\theta, t)$ is governed by the Smoluchowski equation [9]:

$$
\frac{\partial f(\theta, t)}{\partial t}=D_{r} \frac{\partial}{\partial \theta}\left(\frac{\partial V(\theta)}{\partial \theta} f(\theta, t)+\frac{\partial f(\theta, t)}{\partial \theta}\right)
$$

where $D_{r}$ is the rotational diffusion coefficient of polymer rods and $V(\theta)$ the sum of all potentials affecting polymer rods. If the Maier-Saupe potential is the only potential affecting polymer rods, then at equilibrium the orientation distributation of polymer rods is given by the Boltzmann distribution:

$$
f(\theta) \sim \exp \left(-V_{M S}(\theta)\right) .
$$

Notice that when the Maier-Saupe potential is the only potential affecting polymer rods and when only the equilibrium distribution is concerned we can select a suitable coordinate system such that $\langle\sin 2 \theta\rangle=0$. So in this special coordinate system the Maier-Saupe potential (2.1) is simplified to

$$
V_{M S}(\theta)=-U\langle\cos 2 \theta\rangle \cos 2 \theta .
$$

In this special coordinate system, the Maier-Saupe potential is completely specified by a parameter $r$ in the form of $V_{M S}(\theta)=-r \cos 2 \theta$ where $r$ is defined as

$$
r \equiv U\langle\cos 2 \theta\rangle .
$$

In fact, $s \equiv\langle\cos 2 \theta\rangle$ is the Flory order parameter, where $s=0$ corresponds to isotropic phase and $s=1$ describes the fully aligned phase. The equilibrium distribution (2.3) becomes

$$
f(\theta)=\frac{\exp (r \cos 2 \theta)}{\int_{0}^{2 \pi} \exp (r \cos 2 \theta) d \theta} .
$$

The probability density $f(\theta)$ given above depends on $r$. Thus, although (2.5) is the definition of $r$, it is also an equation for $r$ in which $r$ appears on the left side as well as on the right side through the probability density $f(\theta)$. Rewriting equation (2.5) in the form of the order parameter, we have

$$
\langle\cos 2 \theta\rangle=\frac{r}{U} .
$$


Applying integration by parts in the numerator of $\langle\cos 2 \theta\rangle$, one has

$$
\begin{aligned}
\int_{0}^{2 \pi} \cos 2 \theta \exp (r \cos 2 \theta) d \theta & =\frac{1}{2} \int_{0}^{2 \pi} \exp (r \cos 2 \theta) d \sin 2 \theta \\
& =r \int_{0}^{2 \pi} \sin ^{2} 2 \theta \exp (r \cos 2 \theta) d \theta .
\end{aligned}
$$

Substituting into equation (2.7) and dividing by $r$ yields

$$
\left\langle\sin ^{2} 2 \theta\right\rangle=\frac{1}{U}
$$

As an equation for $r,(2.8)$ is equivalent to (2.5) when $r \neq 0$. Equation (2.8) has the advantage that $r$ appears only on the left side of equation. It is straightward to verify that $\left\langle\sin ^{2} 2 \theta\right\rangle_{r=0}=\frac{1}{2}$. In $[7,32]$, it was proved that

$$
\frac{d}{d r}\left\langle\sin ^{2} 2 \theta\right\rangle<0 \text { for } r>0 \text {. }
$$

Therefore, equation (2.8) has a nematic solution $(r>0)$ if and only if $U>2$ [7, 25]. For $U>2$, there is a unique function $r(U)$ such that equation (2.8) is satisfied. Furthermore, $r(U)$ is an increasing function of $U$. This one-to-one correspondence between $U$ and $r$ also allows us to view $U$ as a function of $r$ :

$$
U(r) \equiv \frac{r}{\langle\cos 2 \theta\rangle} \text { or equivalently } U(r) \equiv \frac{1}{\left\langle\sin ^{2} 2 \theta\right\rangle} .
$$

The right-hand side of (2.10) depends on $r$ only. So $U(r)$ can be calculated in a
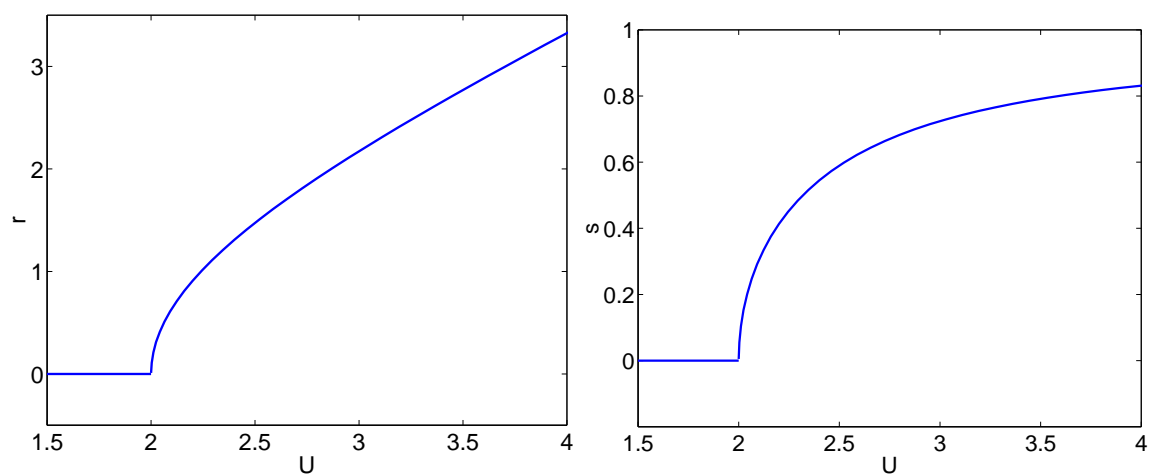

FIG. 2.1. Phase diagrams for the 2D pure nematic polymers (no shear flow). The left panel shows $r$ as a function of the normalized polymer concentration $U$ where $r$ is defined as $r \equiv U\langle\cos 2 \theta\rangle$. Mathematically, it is more convenient to treat $r$ as the independent variable and treat $U$ as a function of $r$. The right panel shows the order parameter $s$ as a function of $U$ where the order parameter is defined as $s \equiv\langle\cos 2 \theta\rangle$. The isotropic-nematic phase transition occurs at $U=2$.

straightforward way without solving non-linear equations. This illustrates that it is mathematically more convenient if we use $r$ as the independent variable although $r$ does not have a clear physical meaning (it is the product of the normalized polymer concentration and the order parameter). Figure 2.1A shows $r$ as a function of $U$ whereas Figure 2.1B depicts the order parameter $s \equiv\langle\cos 2 \theta\rangle$ as a function of $U$. Clearly, the isotropic-nematic phase transition occurs at $U=2$. 


\section{Nematic polymers under an imposed weak shear}

In the presence of an imposed 2-D shear flow $\mathbf{v}=\gamma(0,-x)$, where $\gamma$ is the shear rate, the non-dimensional Peclet number is defined as $\varepsilon \equiv \frac{\gamma}{D_{r}}$ where $D_{r}$ is the rotational diffusion coefficient. Here we use $\varepsilon$ to denote the Peclet number because we study the case of small Peclet number in this paper. Under the imposed shear, the orientation distribution $f(\theta, t)$ evolves according to the Smoluchowski equation [9]:

$$
\frac{\partial f}{\partial t}=D_{r} \frac{\partial}{\partial \theta}\left[\left(-\frac{\varepsilon}{2}+\frac{\varepsilon}{2} V_{S H}^{\prime}(\theta)+V_{M S}^{\prime}(\theta)\right) f+\frac{\partial}{\partial \theta} f\right] .
$$

Recall that $k_{B} T$ has been set to one. The rotational diffusion coefficient $D_{r}$ can be absorbed into a non-dimensional time $\tilde{t}=t D_{r}$. For mathematical simplicity, we set $D_{r}=1$ (or equivalently we use the non-dimensional time). The Maier-Saupe interaction potential $V_{M S}(\theta)$ is given in $(2.1)$ with $k_{B} T=1$. Notice that because of the orientation of imposed shear flow, we no longer have the freedom of selecting the coordinate system to make $\langle\sin 2 \theta\rangle=0$. Instead, we can select an angle $\alpha$ such that

$$
\langle\sin 2(\theta-\alpha)\rangle=0
$$

Expanding $\langle\sin 2 \theta\rangle$ and $\langle\cos 2 \theta\rangle$ in $(2.1)$ yields

$$
V_{M S}(\theta)=-U\langle\cos 2(\theta-\alpha)\rangle \cos 2(\theta-\alpha) .
$$

The potential caused by the shear flow is a tilted periodic function

$$
-\frac{\varepsilon}{2} \theta+\frac{\varepsilon}{2} V_{S H}(\theta)=-\frac{\varepsilon}{2} \theta+\frac{\varepsilon}{2} \cdot \frac{1}{2} \sin 2 \theta,
$$

where $V_{S H}(\theta)=\frac{1}{2} \sin 2 \theta$ is the periodic part of the potential caused by the shear flow, normalized by half of the Peclet number. The case of high shear rate (large Peclet number) was studied in [26, 27] and most recently analyzed in [30]. In this work we consider the case where the imposed shear is weak (small Peclet number), which will allow us to do asymptotic expansions.

We are interested in finding steady state solutions. We first substitute $f(\theta)=$ $f^{(0)}(\theta)+O(\varepsilon)$ into the Smoluchowski equation (3.1) and keep only the leading order terms to obtain

$$
\frac{\partial}{\partial \theta}\left[V_{M S}^{(0)^{\prime}}(\theta) f^{(0)}+\frac{\partial}{\partial \theta} f^{(0)}\right]=0,
$$

where the leading order term of the Maier-Saupe interaction potential is

$$
V_{M S}^{(0)}(\theta)=-U\langle\cos 2(\theta-\alpha)\rangle^{(0)} \cos 2(\theta-\alpha)
$$

Here the notation $\langle\cos 2(\theta-\alpha)\rangle^{(0)}$ means the average of $\cos 2(\theta-\alpha)$ with respect to the leading order term of the probability density $f^{(0)}(\theta)$. (3.6) is simply the governing equation for nematic polymers with only Maier-Saupe interaction potential. In particular, the effect of shear does not appear in the leading term equation (3.6). Thus, for $0<U<2$, we have

$$
f^{(0)}(\theta)=\frac{1}{2 \pi}, \quad V_{M S}^{(0)}(\theta)=0
$$


For $U>2$, there are two steady states [7, 25]: the isotropic state and nematic state. For $U>2$, the isotropic state is unstable [11]; the stable nematic state is

$$
f^{(0)}(\theta)=\frac{\exp (r \cos 2(\theta-\alpha))}{\int_{0}^{2 \pi} \exp (r \cos 2(\theta-\alpha)) d \theta}, \quad V_{M S}^{(0)}(\theta)=-r \cos 2(\theta-\alpha),
$$

where $r>0$ is the solution of equation (2.8). Below we are going to continue with the case of $U>2$ (i.e. the case of $r>0$ ) to see under what condition a steady state exists. Due to the absence of the shear effect in the equation for the leading order terms, the angle $\alpha$ in (3.8) is completely arbitrary and cannot be determined from the leading order equation (3.5). Let us try to determine the angle $\alpha$ from the full equation instead. A solution of the full Smoluchowski equation (3.1) satisfies

$$
\left[-\frac{\varepsilon}{2}+\frac{\varepsilon}{2} V_{S H}^{\prime}(\theta)+V_{M S}^{\prime}(\theta)\right] f+\frac{\partial}{\partial \theta} f=-J(\theta),
$$

where $J(\theta)$ is the probability flux. At a steady state, $J(\theta)=J$ is a constant independent of $\theta$. Multiplying both sides of (3.9) by the integrating factor $\exp \left[-\frac{\varepsilon}{2} \theta+\right.$ $\left.\frac{\varepsilon}{2} V_{S H}(\theta)+V_{M S}(\theta)\right]$ and integrating from $q$ to $q+2 \pi$, we have

$$
\begin{aligned}
& {[1-\exp (-\varepsilon \pi)] \exp \left(-\frac{\varepsilon}{2} q+\frac{\varepsilon}{2} V_{S H}(q)+V_{M S}(q)\right) f(q) } \\
= & J \int_{q}^{q+2 \pi} \exp \left[-\frac{\varepsilon}{2} \theta+\frac{\varepsilon}{2} V_{S H}(\theta)+V_{M S}(\theta)\right] d \theta .
\end{aligned}
$$

Dividing both sides by $J \cdot \exp \left(-\frac{\varepsilon}{2} q+\frac{\varepsilon}{2} V_{S H}(q)+V_{M S}(q)\right)$, expanding the right hand side and using the fact that $V_{M S}(\theta)=V_{M S}^{(0)}(\theta)+O(\varepsilon)$, we obtain

$$
\begin{aligned}
& \frac{[1-\exp (-\varepsilon \pi)]}{J} f(q) \\
= & \exp \left(-V_{M S}^{(0)}(q)\right) \int_{0}^{2 \pi} \exp \left[V_{M S}^{(0)}(\theta)\right] d \theta \cdot(1+O(\varepsilon)) .
\end{aligned}
$$

Integrating both sides from 0 to $2 \pi$ and using that $\int_{0}^{2 \pi} f(q) d q=1$ gives us

$$
\begin{aligned}
& \frac{[1-\exp (-\varepsilon \pi)]}{J} \\
= & \left(\int_{0}^{2 \pi} \exp \left(-V_{M S}^{(0)}(q)\right) d q\right) \cdot\left(\int_{0}^{2 \pi} \exp \left[V_{M S}^{(0)}(\theta)\right] d \theta\right) \cdot(1+O(\varepsilon)) \\
= & 4 \pi^{2} g(r) \cdot(1+O(\varepsilon))
\end{aligned}
$$

In the above, we have used $V_{M S}^{(0)}(\theta)=-r \cos 2(\theta-\alpha)$ and function $g(r)$ is defined as

$$
g(r) \equiv\left(\frac{1}{2 \pi} \int_{0}^{2 \pi} \exp (r \cos 2 \theta) d \theta\right)^{2} .
$$

where $r>0$ is the solution of (2.8). Expanding $1-\exp (-\varepsilon \pi)=\varepsilon \pi \cdot(1+O(\varepsilon))$, we express the probability flux $J$ as

$$
4 \pi J=\frac{\varepsilon}{g(r)} \cdot(1+O(\varepsilon)) .
$$


To find the condition for the existence of steady state, we seek another expression for probability flux $J$. Integrating both sides of (3.9) from 0 to $2 \pi$, we have

$$
-\frac{\varepsilon}{2}+\frac{\varepsilon}{2} \int_{0}^{2 \pi} V_{S H}^{\prime}(\theta) f(\theta) d \theta+\int_{0}^{2 \pi} V_{M S}^{\prime}(\theta) f(\theta) d \theta=-2 \pi J
$$

Let us examine these terms one by one. The third term on the left is,

$$
\begin{aligned}
\int_{0}^{2 \pi} V_{M S}^{\prime}(\theta) f(\theta) d \theta & =\int_{0}^{2 \pi} \frac{d}{d \theta}(-r \cos 2(\theta-\alpha)) f(\theta) d \theta \\
& =\int_{0}^{2 \pi} 2 r \sin 2(\theta-\alpha) f(\theta) d \theta=2 r\langle\sin 2(\theta-\alpha)\rangle=0 .
\end{aligned}
$$

Here we have used the fact that in the Maier-Saupe potential, the angle $\alpha$ is selected to make $\langle\sin 2(\theta-\alpha)\rangle=0$. This result is not surprsing. The term $\int_{0}^{2 \pi} V_{M S}^{\prime}(\theta) f(\theta) d \theta$ is the total force on the ensemble of polymer rods due to the force from the MaierSaupe interaction potential. Since the Maier-Saupe potential is caused by the mutual interaction of polymer rods with each other, Newton's third law guarantees that the total force on the ensemble is zero. The second term on the left of (3.15) is,

$$
\begin{aligned}
\frac{\varepsilon}{2} \int_{0}^{2 \pi} V_{S H}^{\prime}(\theta) f(\theta) d \theta & =\frac{\varepsilon}{2} \int_{0}^{2 \pi} \cos 2 \theta f(\theta) d \theta \\
& =\frac{\varepsilon}{2}\langle\cos 2 \theta\rangle=\frac{\varepsilon}{2}\langle\cos 2(\theta-\alpha) \cos 2 \alpha-\sin 2(\theta-\alpha) \sin 2 \alpha\rangle \\
& =\frac{\varepsilon}{2}\langle\cos 2(\theta-\alpha) \cos 2 \alpha\rangle=\frac{\varepsilon}{2}\left[\langle\cos 2(\theta-\alpha)\rangle^{(0)} \cos 2 \alpha+O(\varepsilon)\right] \\
& =\frac{\varepsilon}{2}\left[\frac{r}{U} \cos 2 \alpha+O(\varepsilon)\right] .
\end{aligned}
$$

Here again we used $\langle\sin 2(\theta-\alpha)\rangle=0$. Substituting the results (3.16) and (3.17) into (3.15) yields another expression for the probability flux $J$

$$
4 \pi J=\varepsilon \cdot\left[1-\frac{r}{U} \cos 2 \alpha+O(\varepsilon)\right] .
$$

Equating (3.14) with (3.18), we obtain

$$
\frac{1}{g(r)}=1-\frac{r}{U} \cos 2 \alpha
$$

(3.19) is the equation for determining the angle $\alpha$. Here mathematically we treat $r$ as an independent variable and treat others as functions of $r$. In particular, $U$ is a function of $r$ (although from physical point of view, it is more reasonable to treat $U$ as an independent variable and treat $r$ as a function of $U$ ). Now we investigate for what values of $r$ equation (3.19) has a solution and for what values of $r$ it does not. Writing $\cos 2 \alpha=1-2 \sin ^{2} \alpha$ and rearranging terms, we arrive at

$$
2 \sin ^{2} \alpha=\frac{h(r)}{g(r)},
$$

where function $h(r)$ is defined as

$$
h(r) \equiv \frac{U}{r}\left[1-g(r)\left(1-\frac{r}{U}\right)\right] .
$$


The properties of $h(r)$ and $g(r)$ are described in the theorem below.

THEOREM 3.1. For function $g(r)$ defined in (3.13) and function $h(r)$ defined in (3.21), we have

1. $g(0)=1$ and $g(r)>0$.

2. $g^{\prime}(r)>0$ for $r>0$, which implies that $g(r)>1$ for $r>0$.

3. $h(0)=1$ and $h^{\prime}(0)=-1<0$.

4. $\lim _{r \rightarrow \infty} h(r)=-\infty$.

The proof of Theorem 3.1 is given in Appendix A.
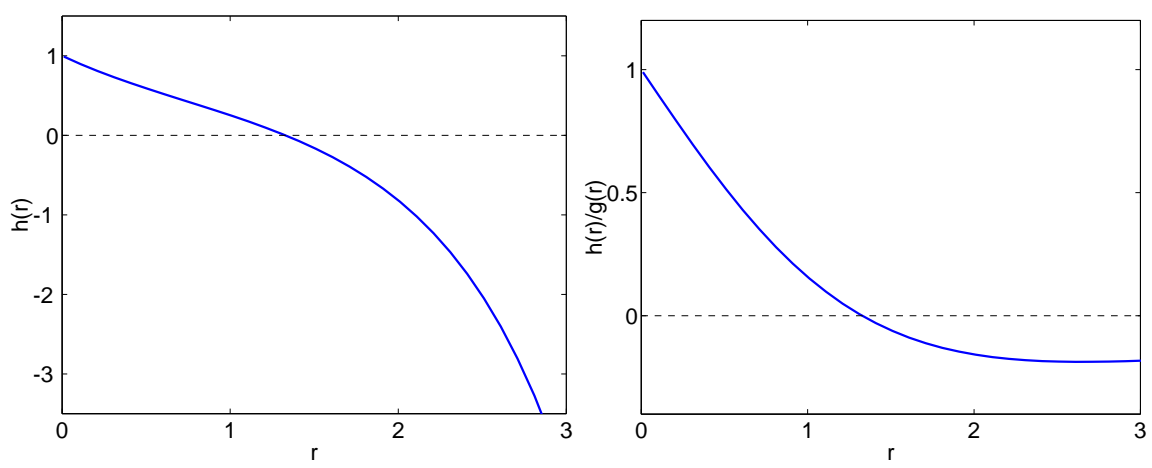

FIG. 3.1. Graphs of functions $h(r)$ and $\frac{h(r)}{g(r)}$. The left panel shows the graph of function $h(r)$. From the graph, it is clear that $h(r)$ is a decreasing function of $r$ for $r>0$ and $h(r) \rightarrow-\infty$ as $r \rightarrow+\infty$, as described in Theorem 1. $h(r)$ intersects with the horizontal axis at $r_{0}=1.32757384$. Function $h(r)$ satisfies $1>h(r)>0$ for $r<r_{0}$ and $h(r)<0$ for $r>r_{0}$. The right panel shows the graph of function $\frac{h(r)}{g(r)}$.

The graph on the left in Figure 3.1 indicates that $h(r)$ is a decreasing function of $r$ for $r>0$. A rigorous mathematical proof for this conclusion, however, is still open. Since $h(0)=1, h(r)$ is a decreasing function of $r$ for $r>0$ and $\lim _{r \rightarrow \infty} h(r)=-\infty$, there exists a unique positive number $r_{0}$ such that

$$
\begin{aligned}
1>h(r)>0 & \text { for } \quad r<r_{0}, \\
h(r)<0 & \text { for } \quad r>r_{0} .
\end{aligned}
$$

Using the property that $g(0)=1$ and $g(r)>1$ for $r>0$, we see that the right side of equation (3.20) has the behavior

$$
\begin{aligned}
1> & \frac{h(r)}{g(r)}>0 \quad \text { for } \quad r<r_{0}, \\
\frac{h(r)}{g(r)}<0 \quad & \text { for } \quad r>r_{0} .
\end{aligned}
$$

This behavior of $\frac{h(r)}{g(r)}$ is shown in the graph on the right in Figure 3.1. Let $U_{0} \equiv U\left(r_{0}\right)$ be the value of $U$ corresponding to $r=r_{0}$ where function $U(r)$ is defined in (2.10). Since $U(r)$ is an increasing function of $r$, the region of $U<U_{0}$ corresponds to the region of $r<r_{0}$. 
Now let us get back to solving equation (3.20). From the behavior of $\frac{h(r)}{g(r)}$ obtained above, it is clear that when $U<U_{0}$, a steady state solution exists. When $U>U_{0}$, there is no steady state solution. The numerical values of $r_{0}, U_{0}$ and the corresponding order parameter can be calculated using numerical integration and are listed below:

$$
\begin{aligned}
& r_{0}=1.32757384, \\
& U_{0} \equiv U\left(r_{0}\right)=2.41144646, \\
& s_{0} \equiv \frac{r_{0}}{U_{0}}=0.550530090 .
\end{aligned}
$$

Our value of $U_{0}$ is consistent with the one found in $[26,27]$.

4. Multi-scale solution of the Smoluchowski equation for nematic polymers under a weak shear

The analysis in the previous section shows that under an imposed weak shear when the normalized polymer concentration $U$ is above the threshold $U_{0}$ there is no steady state solution. To study the unsteady state dynamics of the probability density for the case of $U>U_{0}$ and to study the convergence of the probability density to steady state for the case of $U<U_{0}$, we carry out multi-scale asymptotic expansion $[2,21]$ of the Smoluchowski equation (3.1). The approach of multi-scale expansions has been applied to study the nonlinear dynamics of a nematic liquid crystal under a shear flow for a Landau-de Gennes model [28] and a Doi-Hess mesoscopic orientation tensor model [3]. Our goal here is to capture the slow time evolution of the probability density governed by the Smoluchowski equation.

Let $T_{0}=t$ and $T_{1}=\varepsilon \cdot t$. We seek an expansion of the form

$$
f(\theta, t)=f^{(0)}\left(\theta, T_{0}, T_{1}\right)+\varepsilon f^{(1)}\left(\theta, T_{0}, T_{1}\right)+O\left(\varepsilon^{2}\right) .
$$

Substituting the multi-scale expansion (4.1) into the Smoluchowski equation (3.1) and keeping only the $O(1)$ terms, we have

$$
\frac{\partial f^{(0)}}{\partial T_{0}}=\frac{\partial}{\partial \theta}\left[V_{M S}^{(0)^{\prime}} f^{(0)}+\frac{\partial}{\partial \theta} f^{(0)}\right] .
$$

This is exactly the governing equation for nematic polymers in the absence of shear flow. Steady state solutions of (4.2) are equilibria and are given by the Boltzmann distribution

$$
f^{(0)}\left(\theta, T_{0}, T_{1}\right)=\frac{\exp (r \cos 2(\theta-\alpha))}{\int_{0}^{2 \pi} \exp (r \cos 2(\theta-\alpha)) d \theta} .
$$

Again, mathematically it is more convenient if we simply treat $r$ as an independent variable. If we choose to treat $U$ as the independent variable and view $r$ as a function of $U$, then $r(U)$ can be solved from equation (2.8). Suppose the polymer orientation starts with the equilibrium distribution given in (4.3). Equation (4.2) dictates that the polymer orientation distribution stays unchanged with respect to $T_{0}$. That is,

$$
\frac{\partial f^{(0)}\left(\theta, T_{0}, T_{1}\right)}{\partial T_{0}}=0 .
$$

We say that $f^{(0)}\left(\theta, T_{0}, T_{1}\right)$ is a quasi-steady state if it is independent of $T_{0}$. In particular, the phase angle $\alpha$ in (4.3) is independent of $T_{0}$. However, as we will see, the phase 
angle $\alpha$ may evolve with $T_{1}$, which corresponds to the slow time evolution caused by the weak shear flow. To calculate $\alpha\left(T_{1}\right)$, we look at the expansion of $\frac{\partial f(\theta, t)}{\partial t}$ :

$$
\frac{\partial f(\theta, t)}{\partial t}=\frac{\partial f^{(0)}\left(\theta, T_{0}, T_{1}\right)}{\partial T_{0}}+\varepsilon\left[\frac{\partial f^{(0)}\left(\theta, T_{0}, T_{1}\right)}{\partial T_{1}}+\frac{\partial f^{(1)}\left(\theta, T_{0}, T_{1}\right)}{\partial T_{0}}\right]+\cdots
$$

If the polymers start with an equilibrium distribution for pure nematic polymers (no shear), then $f^{(0)}\left(\theta, T_{0}, T_{1}\right)$ is a quasi-steady state and the $O(1)$ term in the expansion of $\frac{\partial f(\theta, t)}{\partial t}$ is zero. The $O(\varepsilon)$ term in $\frac{\partial f(\theta, t)}{\partial t}$ contains two parts. In multi-scale expansions, to separate the effects of time scales $T_{0}$ and $T_{1}$, it is necessary to impose condition(s) on the $f^{(1)}\left(\theta, T_{0}, T_{1}\right)$ term. Here we impose the condition that $f^{(1)}\left(\theta, T_{0}, T_{1}\right)$ is also a quasi-steady state (that is, it is independent of $T_{0}$ ). From physical point of view, it is reasonable to impose the condition that $f^{(1)}\left(\theta, T_{0}, T_{1}\right)$ is a quasi-steady state. In the absence of shear flow, the Maier-Saupe interaction potential drives the polymers to the equilibrium orientation distribution. The effect of imposed weak shear flow will disturb the equilibrium distribution. Since the imposed shear is weak the perturbation on orientation distribution is also small. More specifically, during a time period of $\tau$, the total perturbation on the orientation distribution is of the order $\varepsilon \tau$. During the time period of $\tau$, the orientation distribution will constantly try to relax to new equilibriums. As we discussed above, the leading term of the orientation distribution is in a quasi-steady state (independent of $T_{0}$ ). The leading term of the orientation distribution rotates with respect to the slow time scale $T_{1}$. In a time period of $\tau$, the leading term of the orientation distribution rotates by $\varepsilon \tau$. Here it is important to point out that the Maier-Saupe interaction potential rotates along with the orientation distribution. So if we rotate the coordinate system to follow the leading term of the orientation distribution, then the leading term of the Maier-Saupe potential will be stationary. In the rotating coordinate system, the effect of the weak shear also changes at most by $O(\varepsilon \cdot \varepsilon \tau)=O\left(\varepsilon^{2} \tau\right)$. As a result, in the rotating coordinate system, the orientation distribution can change at most by $O\left(\varepsilon^{2} \tau\right)$ over the time period of $\tau$. Therefore, in the rotating coordinate system, the rate of change of the orientation distribution is of the order $O\left(\varepsilon^{2}\right)$, which implies that $f^{(1)}\left(\theta, T_{0}, T_{1}\right)$ is independent of the fast time $T_{0}$. It has also been verified in our extensive numerical simulations (results not shown) that $f^{(1)}\left(\theta, T_{0}, T_{1}\right)$ is a quasi-steady state.

Since $f^{(1)}\left(\theta, T_{0}, T_{1}\right)$ is independent of $T_{0}$, the expansion of $\frac{\partial f(\theta, t)}{\partial t}$ becomes

$$
\frac{\partial f(\theta, t)}{\partial t}=\varepsilon \frac{\partial f^{(0)}\left(\theta, T_{0}, T_{1}\right)}{\partial T_{1}}+O\left(\varepsilon^{2}\right)
$$

Using the Boltzmann form of $f^{(0)}\left(\theta, T_{0}, T_{1}\right)$ given in (4.3), we have

$$
\frac{\partial f(\theta, t)}{\partial t}=-\varepsilon \frac{\partial f^{(0)}\left(\theta, T_{0}, T_{1}\right)}{\partial \theta} \cdot \frac{d \alpha\left(T_{1}\right)}{d T_{1}}+O\left(\varepsilon^{2}\right) .
$$

Substituting the expansion of $\frac{\partial f(\theta, t)}{\partial t}$ into the Smoluchowski equation (3.1), moving all terms to the right side and using the fact that $\alpha$ is independent of $\theta$ ( $\alpha$ depends only on $T_{1}$ ), we obtain

$$
0=\frac{\partial}{\partial \theta}\left[\left(-\frac{\varepsilon}{2}+\frac{\varepsilon}{2} V_{S H}^{\prime}(\theta)+V_{M S}^{\prime}(\theta)\right) f(\theta)+\frac{\partial f(\theta)}{\partial \theta}+\varepsilon f^{(0)}(\theta) \frac{d \alpha\left(T_{1}\right)}{d T_{1}}+O\left(\varepsilon^{2}\right)\right]
$$

It follows that

$$
\left(-\frac{\varepsilon}{2}+\frac{\varepsilon}{2} V_{S H}^{\prime}(\theta)+V_{M S}^{\prime}(\theta)\right) f(\theta)+\frac{\partial f(\theta)}{\partial \theta}+\varepsilon f^{(0)}(\theta) \frac{d \alpha\left(T_{1}\right)}{d T_{1}}+O\left(\varepsilon^{2}\right)=-\varepsilon J_{2}
$$


where $\varepsilon J_{2}$ is the $O(\varepsilon)$ term of the integration constant, independent of $\theta$. Since the solution of the $O(1)$ term equation (4.2) is an equilibrium, the $O(1)$ term of the integration constant is zero.

In (4.7), both $\frac{d \alpha\left(T_{1}\right)}{d T_{1}}$ and $J_{2}$ are unknown. We need to solve for both of them simultaneously. For that purpose, we need two linear equations for $\frac{d \alpha\left(T_{1}\right)}{d T_{1}}$ and $J_{2}$. Integrating (4.7) from 0 to $2 \pi$ and dividing by $\varepsilon$ yields

$$
-\frac{1}{2}+\frac{1}{2} \frac{r}{U} \cos \left(2 \alpha\left(T_{1}\right)\right)+\frac{d \alpha\left(T_{1}\right)}{d T_{1}}+O(\varepsilon)=-2 \pi J_{2} .
$$

In the integration above we have used results (3.16) and (3.17). The second linear equation for $\frac{d \alpha\left(T_{1}\right)}{d T_{1}}$ and $J_{2}$ is obtained by dividing (4.7) by $\varepsilon f(\theta)$, approximating $f(\theta)$ by $f^{(0)}$ and then integrating from 0 and $2 \pi$ :

$$
-\pi+2 \pi \frac{d \alpha\left(T_{1}\right)}{d T_{1}}+O(\varepsilon)=-(2 \pi)^{2} g(r) J_{2} .
$$

Here we have used the fact that

$$
\begin{aligned}
& \int_{0}^{2 \pi} \frac{1}{f^{(0)}(\theta)} d \theta=\left(\int_{0}^{2 \pi} \exp (r \cos 2(\theta-\alpha)) d \theta\right)\left(\int_{0}^{2 \pi} \exp (-r \cos 2(\theta-\alpha)) d \theta\right) \\
& =(2 \pi)^{2} g(r) .
\end{aligned}
$$

where function $g(r)$ is defined in (3.13). Solving $\frac{d \alpha\left(T_{1}\right)}{d T_{1}}$ from (4.8) and (4.9) yields

$$
\frac{d \alpha\left(T_{1}\right)}{d T_{1}}=\frac{1}{2}\left[1-\frac{g(r)}{g(r)-1} \cdot \frac{r}{U} \cos \left(2 \alpha\left(T_{1}\right)\right)\right] .
$$

Writing $\cos \left(2 \alpha\left(T_{1}\right)\right)=1-2 \sin ^{2} \alpha\left(T_{1}\right)$, we obtain

$$
\frac{d \alpha\left(T_{1}\right)}{d T_{1}}=\frac{g(r)}{g(r)-1} \cdot \frac{r}{U}\left[\sin ^{2}\left(\alpha\left(T_{1}\right)\right)-\frac{h(r)}{2 g(r)}\right],
$$

where function $h(r)$ is defined in (3.21). The slow time evolution of the phase angle $\alpha\left(T_{1}\right)$ is governed by the differential equation (4.12). In equation (4.12), the factor $\frac{g(r)}{g(r)-1} \cdot \frac{r}{U}$ is always positive (for $U>2$ ). As we discussed in the previous section, for $U<U_{0}=2.41144646$, we have $0<\frac{h(r)}{2 g(r)}<0.5$ and $\alpha\left(T_{1}\right)$ will converge to a steady state angle $\alpha^{(S)}$ satisfying

$$
\sin ^{2}\left(\alpha^{(S)}\right)=\frac{h(r)}{2 g(r)} .
$$

There are two angles satisfying (4.13) in the interval $\left[-\frac{\pi}{2}, \frac{\pi}{2}\right]$, one positive and one negative. The whole problem is periodic with period $\pi$ so we only need to examine an interval of size $\pi$. The stability of a steady state angle $\alpha^{(S)}$ is determined by expanding equation (4.12) around $\alpha^{(S)}$. Near $\alpha^{(S)}$, we have

$$
\frac{d\left(\alpha\left(T_{1}\right)-\alpha^{(S)}\right)}{d T_{1}}=\frac{g(r)}{g(r)-1} \cdot \frac{r}{U} \cdot \sin \left(2 \alpha^{(S)}\right)\left(\alpha\left(T_{1}\right)-\alpha^{(S)}\right)
$$

It follows that $\alpha^{(S)}$ is stable if $\sin \left(2 \alpha^{(S)}\right)<0$ and $\alpha^{(S)}$ is unstable if $\sin \left(2 \alpha^{(S)}\right)>0$. So the positive steady state angle is unstable whereas the negative steady state angle is stable. 
When $U>U_{0}=2.41144646$, we have $\frac{h(r)}{2 g(r)}<0$ and $\alpha\left(T_{1}\right)$ will keep increasing with $T_{1}$ because the right side of (4.12) is always positive. So when $U>U_{0}=2.41144646$, the director will keep rotating (tumbling) and there is no steady state. In the following we derive the period of the tumbling state.

Let $\tau_{p}$ be the period of tumbling in slow time $T_{1} \equiv \varepsilon t$. For $U>U_{0}=2.41144646$, differential equation (4.12) has the form

$$
\frac{d \alpha}{d T_{1}}=c_{1}\left(\sin ^{2} \alpha+c_{2}\right), c_{1} \equiv \frac{g(r)}{g(r)-1} \cdot \frac{r}{U}>0, c_{2} \equiv \frac{-h(r)}{2 g(r)}>0 .
$$

Consider the period $\left[\tau_{0}, \tau_{0}+\tau_{p}\right]$ in $T_{1}$ that corresponds to $\alpha$ going from 0 to $2 \pi$. Dividing both sides of (4.14) by $\left(\sin ^{2} \alpha+c_{2}\right)$ and integrating over the period $\left[\tau_{0}, \tau_{0}+\tau_{p}\right]$ yields

$$
\int_{0}^{2 \pi} \frac{1}{\sin ^{2} \alpha+c_{2}} d \alpha=c_{1} \tau_{p}
$$

Because of the symmetry of function $\sin ^{2} \alpha$, on the left side of (4.15), the integral over $[0,2 \pi]$ is equal to 4 times the integral over $[0, \pi / 2]$. Using substitution $s=\tan (\alpha)$, we obtain

$$
4 \int_{0}^{\infty} \frac{1}{\left(1+c_{2}\right) s^{2}+c_{2}} d s=c_{1} \tau_{p} .
$$

Applying substitution $u=s \sqrt{\left(1+c_{2}\right) / c_{2}}$ and using the fact that $\int_{0}^{\infty} \frac{1}{u^{2}+1} d u=\frac{\pi}{2}$, we arrive at

$$
\tau_{p}=2 \pi \frac{1}{c_{1} \sqrt{c_{2}\left(1+c_{2}\right)}}
$$

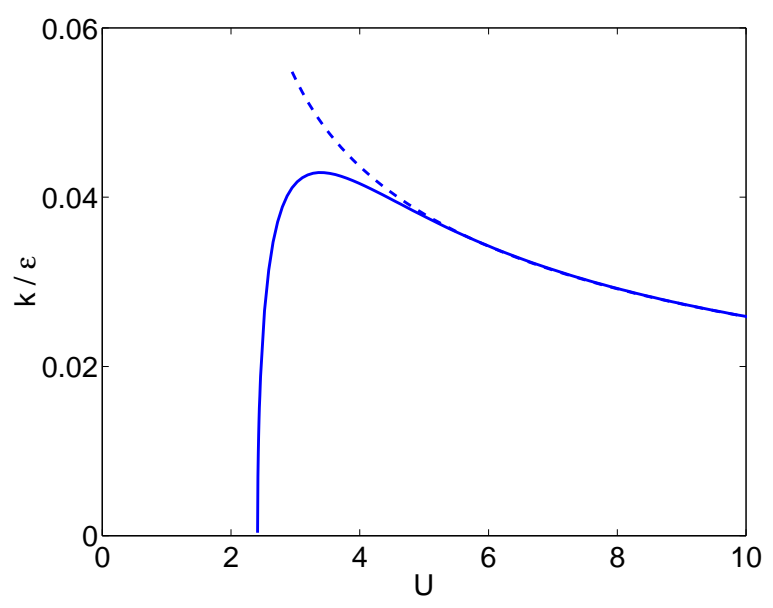

FIG. 4.1. Solid line shows the normalized tumbling frequency $(k / \varepsilon)$ as a function of $U$. The dashed line shows the asymptotic approximation for the normalized tumbling frequency as $U \rightarrow \infty$.

Thus, in time $t$, the period of tumbling is given by

$$
t_{p}=\frac{2 \pi}{\varepsilon} \cdot \frac{1}{\left(\frac{g(r)}{g(r)-1} \cdot \frac{r}{U}\right) \sqrt{\left(\frac{-h(r)}{2 g(r)}\right)\left[1+\left(\frac{-h(r)}{2 g(r)}\right)\right]}}
$$


The tumbling frequency $(k)$ normalized by the Peclet number $(\varepsilon)$ is

$$
\frac{k}{\varepsilon}=\frac{1}{t_{p} \varepsilon}=\frac{1}{2 \pi} \cdot\left(\frac{g(r)}{g(r)-1} \cdot \frac{r}{U}\right) \sqrt{\left(\frac{-h(r)}{2 g(r)}\right)\left[1+\left(\frac{-h(r)}{2 g(r)}\right)\right]} .
$$

In Figure 4.1, the solid line shows the normalized tumbling frequency $(k / \varepsilon)$ as a function of $U$. As $U$ increases from $U_{0}$, the normalized tumbling frequency increases steeply to reach a maximum value. Beyond the location where the maximum value is attained, when $U$ increases further to infinity, the normalized tumbling frequency decreases monotonically to zero. As $U \rightarrow \infty$, the order parameter satisfies $\langle\cos 2 \theta\rangle=$ $r / U \rightarrow 1$, which implies $r \rightarrow \infty$. In Appendix A, we show that as $r \rightarrow \infty$ functions $g(r)$ and $h(r)$ have asymptotic expansions $g(r)=1 /(2 \pi r) \exp (2 r)(1+\cdots)$ and $h(r)=$ $-1 /\left(4 \pi r^{2}\right) \exp (2 r)(1+\cdots)$. Substituting these results into (4.19), we arrive at an asymptotic approximation for the normalized tumbling frequency as $U \rightarrow \infty$.

$$
\frac{k}{\varepsilon}=\frac{1}{4 \pi} \cdot \frac{1}{\sqrt{r}}+\cdots \quad \text { as } U \rightarrow+\infty .
$$

In Figure 4.1, the dashed line shows the asymptotic approximation for the normalized tumbling frequency as $U \rightarrow \infty$. As shown in the figure, the asymptotic approximation is very close to the true value for $U>4$.

In summary, the leading term expansion of the solution of Smoluchowski equation (3.1) is given by

$$
f^{(0)}(\theta, t)=\frac{\exp [r \cos 2(\theta-\alpha(\varepsilon t))]}{\int_{0}^{2 \pi} \exp [r \cos 2(\theta-\alpha(\varepsilon t))] d \theta},
$$

where the anglular coordinate of the director $\alpha\left(T_{1}\right)$ is governed by the differential equation (4.12). For $U<U_{0}$, the director converges to a stationary angle and we have a steady state. For $U>U_{0}$, the director keeps rotating and we have a tumbling state. For 2D nematic polymers under an imposed weak shear, these are the only two possible states. In particular, there is no transient oscillatory (wagging) solution.

\section{Behavior of the Smoluchowski equation near $U=2$}

As discussed in Section 2, in the absence of shear flow, the isotropic to nematic phase transition occurs at $U=2$. For fixed $U<2$, as $\varepsilon \rightarrow 0$, the polymer orientation distribution converges to the isotropic phase. For fixed $U>2$, as $\varepsilon \rightarrow 0$, the orientation distribution converges to the nematic phase. Now we study the behavior near $U=2$ when $\varepsilon$ is small but is finite.

Notice, in particular, that in the region near $U=2$ we have $U<U_{0}$ and consequently the polymer orientation distribution has a steady state. Here again, it is mathematically more convenient to treat $r$ as the independent variable. Below we investigate the relation between $U$ and $r$ near $r=0$ in the steady state. More specifically, we study the region where $r$ is the range of $\left[0, o\left(\varepsilon^{\frac{1}{4}}\right)\right]$. In the proof of Theorem 1 given in Appendix A, we showed $g(0)=h(0)=1$, which implies $\lim _{r \rightarrow 0} \frac{h(r)}{2 g(r)}=0.5$. It follows that when $r$ is small, the stable steady state phase angle determined by equation (4.13) is approximately $\alpha=-\frac{\pi}{4}$ (the unstable steady state phase angle is approximately $\alpha=\frac{\pi}{4}$ ). Substituting the phase angle $\alpha$ into the Maier-Saupe interaction potential, we have

$$
-r \cos 2(\theta-\alpha)=-r \cos \left(2 \theta+\frac{\pi}{2}\right)=r \sin 2 \theta .
$$


That is, when $r$ is small, the periodic part of the potential caused by the shear flow is aligned with the Maier-Saupe potential. The sum of these two potentials is

$$
\frac{\varepsilon}{2} V_{S H}(\theta)+V_{M S}(\theta)=-\left(\frac{\varepsilon}{4}+r\right) \cos 2(\theta-\alpha) .
$$

If we treat $r$ as an independent variable, $U$ as a function of $r$ is given by

$$
U_{\varepsilon}(r) \equiv \frac{r}{\langle\cos 2(\theta-\alpha)\rangle_{\varepsilon}}
$$

The subscript in $U_{\varepsilon}(r)$ indicates that it is for the case where the shear flow is present and the effect of shear flow is included in calculating the average. The inverse function $r_{\varepsilon}(U)$ is probably more meaningful from the physical point of view and the order parameter $s$ as a function of the normalized polymer concentration $U$ is given by $s(U) \equiv \frac{r_{\varepsilon}(U)}{U}$. For small $r$, it is necessary to include the effect of shear flow in the leading order expansion. In (5.1), when $r$ is small, $\varepsilon$ may not be ignored and may even be the dominant term relative to $r$. To include the effect of shear flow, we start by finding a more accurate expansion for the steady state probability density $f(\theta)$. Note that in the presence of shear flow, the steady state probability density is no longer given by the Boltzmann distribution. Fortunately, $f(\theta)$ can be expressed from equation (3.10) in the form:

$$
f(\theta)=c \cdot \frac{\int_{0}^{2 \pi} \exp \left[-\frac{\varepsilon}{2} \omega+\frac{\varepsilon}{2} V_{S H}(\omega+\theta)+V_{M S}(\omega+\theta)\right] d \omega}{\exp \left(\frac{\varepsilon}{2} V_{S H}(\theta)+V_{M S}(\theta)\right)} .
$$

Here we have absorbed into the constant $c$ all factors that are independent of $\theta$. Notice that (3.10) is exact so (5.2) is also exact. Now let us expand the integral in (5.2) and keep terms up to $O(\varepsilon)$. Remember that $r$ is in the range of $\left[0, o\left(\varepsilon^{\frac{1}{4}}\right)\right]$.

$$
\begin{aligned}
& \int_{0}^{2 \pi} \exp \left[-\frac{\varepsilon}{2} \omega+\frac{\varepsilon}{2} V_{S H}(\omega+\theta)+V_{M S}(\omega+\theta)\right] d \omega \\
= & \int_{0}^{2 \pi} \exp \left[-\frac{\varepsilon}{2} \omega-\left(\frac{\varepsilon}{4}+r\right) \cos 2(\omega+\theta-\alpha)\right] d \omega \\
= & \int_{0}^{2 \pi}\left[1-\frac{\varepsilon}{2} \omega-\varepsilon\left(\frac{\varepsilon}{4}+r\right) \omega \cos 2(\omega+\theta-\alpha)-\left(\frac{\varepsilon}{4}+r\right)^{2} \cos ^{2} 2(\omega+\theta-\alpha)+\cdots\right] d \omega .
\end{aligned}
$$

In the above, only the third term may depend on $\theta$ but it is of the order $\varepsilon \cdot O\left(\frac{\varepsilon}{4}+\right.$ $r)=o\left(\varepsilon^{\frac{5}{4}}\right)$. All other terms are independent of $\theta$. In particular, the fourth term is independent of $\theta$ and is of the order $O\left(\left(\frac{\varepsilon}{4}+r\right)^{2}\right)=o\left(\varepsilon^{\frac{1}{2}}\right)$. Thus, accurate up to $o\left(\varepsilon^{\frac{5}{4}}\right)$ the integral in (5.2) is independent of $\theta$. In (5.2), absorbing the integral into the constant $c$, we obtain

$$
\begin{aligned}
f(\theta) & =c \cdot \exp \left(-\frac{\varepsilon}{2} V_{S H}(\theta)-V_{M S}(\theta)\right)+o\left(\varepsilon^{\frac{5}{4}}\right) \\
& =\frac{\exp \left[\left(\frac{\varepsilon}{4}+r\right) \cos 2(\theta-\alpha)\right]}{\int_{0}^{2 \pi} \exp \left[\left(\frac{\varepsilon}{4}+r\right) \cos 2(\theta-\alpha)\right] d \theta}+o\left(\varepsilon^{\frac{5}{4}}\right) .
\end{aligned}
$$

Comparing the probability density in (5.3) with the probability density in the case of no shear flow given in (2.6), we see that the only difference is that $r$ in (2.6) is replaced by $\left(\frac{\varepsilon}{4}+r\right)$ in (5.3). As a result, the order parameter in the presence of shear 
flow is equal to the order parameter in the absence of shear flow but with $r$ replaced by $\left(\frac{\varepsilon}{4}+r\right)$ when calculating the average. Mathematically, we have

$$
\langle\cos 2(\theta-\alpha)\rangle_{\varepsilon}=\left.\langle\cos 2(\theta-\alpha)\rangle\right|_{r \leftarrow\left(\frac{\varepsilon}{4}+r\right)} .
$$

Using $\frac{r}{U_{\varepsilon}(r)}=\langle\cos 2(\theta-\alpha)\rangle_{\varepsilon}$ and $\frac{r}{U(r)}=\langle\cos 2(\theta-\alpha)\rangle$, it follows that

$$
\frac{r}{U_{\varepsilon}(r)}=\frac{\frac{\varepsilon}{4}+r}{U\left(\frac{\varepsilon}{4}+r\right)} \text {. }
$$

Writing $U_{\varepsilon}(r)$ as a function of $r$, we arrive at

$$
U_{\varepsilon}(r)=\frac{r}{\frac{\varepsilon}{4}+r} \cdot U\left(\frac{\varepsilon}{4}+r\right),
$$

where function $U_{\varepsilon}(r)$ is for the case with shear flow and function $U(r)$ is for the case of no shear flow. Relation (5.6) shows that the function $U_{\varepsilon}(r)$ can be obtained by a simple algebraic transform on the function $U(r)$. Relation (5.6) is also valid when $r$ is not small. When $r$ is finite and as $\varepsilon \rightarrow 0$, (5.6) simply tells us that $U_{\varepsilon}(r) \approx U(r)$, which is true and is what we obtained in our leading term expansion. Therefore, relation (5.6) is valid for the full range of $r$.

The phase diagram can be expressed in terms of the inverse function of $U_{\varepsilon}(r)$ :

$$
s_{\varepsilon}(U) \equiv \frac{r_{\varepsilon}(U)}{U} .
$$

Phase diagrams for $\varepsilon=0$ (no shear) and $\varepsilon=0.1$ (weak shear) are shown in Figure 5.1. There are several regimes in the phase diagram. To analyze these regimes, we need the expansion of function $U(r)$ around $r=0$ :

$$
U(r)=2\left(1+\frac{1}{8} r^{2}+O\left(r^{4}\right)\right)
$$

Regime 1: $r$ is in the range of $[0, O(\sqrt{\varepsilon})]$.

In this case, expanding $U\left(\frac{\varepsilon}{4}+r\right)$ in (5.6) yields

$$
U_{\varepsilon}(r)=\frac{r}{\frac{\varepsilon}{4}+r} \cdot 2(1+O(\varepsilon))
$$

which indicates that the corresponding range for $U_{\varepsilon}$ is $[0,2-O(\sqrt{\varepsilon})]$. In $(5.8)$, neglecting the $O(\varepsilon)$ term and expressing $r$ as a function of $U_{\varepsilon}$, we have

$$
r_{\varepsilon}(U)=\frac{\varepsilon}{4} \cdot \frac{U}{2-U}, \quad s_{\varepsilon}(U) \equiv \frac{r_{\varepsilon}(U)}{U}=\frac{\varepsilon}{4} \cdot \frac{1}{2-U} .
$$

(5.9) is valid for $U$ in the range of $[0,2-O(\sqrt{\varepsilon})]$. In particular, we have $s_{\varepsilon}(0)=\frac{\varepsilon}{8}$. In other words, the order parameter is non-zero even when the normalized polymer concentration $U$ goes to zero. Here the alignment is caused by the imposed shear flow.

Regime 2: $r$ is of the order of $O\left(\varepsilon^{1 / 3}\right)$.

In this case, expanding $U\left(\frac{\varepsilon}{4}+r\right)$ in (5.6) yields

$$
U_{\varepsilon}(r)=\frac{r}{\frac{\varepsilon}{4}+r} \cdot 2\left(1+\frac{1}{8} r^{2}+O\left(\varepsilon^{4 / 3}\right)\right),
$$



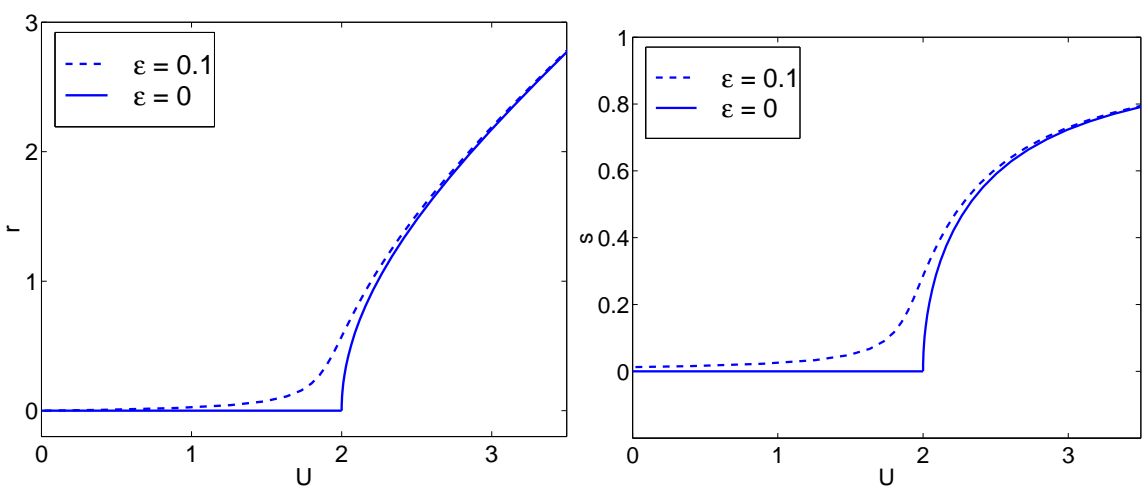

FIG. 5.1. Phase diagrams for the 2D nematic polymers with and without shear flow. $\varepsilon$ is the Peclet number. The left panel shows $r$ as a function of the normalized polymer concentration $U$ where $r$ is defined as $r \equiv U\langle\cos 2 \theta\rangle$. The right panel shows the order parameter $s$ as a function of $U$ where the order parameter is defined as $s \equiv\langle\cos 2 \theta\rangle$. In the absence of shear flow, the isotropicnematic phase transition occurs at $U=2$. In the presence of shear flow, the phase transition occurs gradually over a region near $U=2$.

which indicates that the corresponding range for $U_{\varepsilon}$ is $\left[2-O\left(\varepsilon^{2 / 3}\right), 2+O\left(\varepsilon^{2 / 3}\right)\right]$ and the corresponding order parameter is of the order of $O\left(\varepsilon^{1 / 3}\right)$. From (5.10), the order parameter $s$ can be related to the normalized polymer concentration $U$ through a cubic equation. In (5.10), dividing both sides by $U$ and using $r=U \cdot s$ yields

$$
1=\frac{s}{\frac{\varepsilon}{4}+U \cdot s} \cdot 2\left(1+\frac{U^{2}}{8} s^{2}\right) .
$$

Using $s=O\left(\varepsilon^{1 / 3}\right)$ and $U=2+O\left(\varepsilon^{2 / 3}\right)$ to write $1+\frac{U^{2}}{8} s^{2}=1+\frac{1}{2} s^{2}+O\left(\varepsilon^{4 / 3}\right)$, neglecting the $O\left(\varepsilon^{4 / 3}\right)$ term, and then solving $U$ in terms of s, we arrive at

$$
U_{\varepsilon}(s)=2+\left(1-\frac{\varepsilon}{4 s^{3}}\right) s^{2} .
$$

The inverse function $s_{\varepsilon}(U)$ can be obtained by solving cubic equation (5.11):

$$
s_{\varepsilon}(U)=\left(\frac{\varepsilon}{4}\right)^{1 / 3}\left[\left(\frac{1}{2}+\sqrt{\frac{1}{4}-q^{3}}\right)^{1 / 3}+\frac{q}{\left(\frac{1}{2}+\sqrt{\frac{1}{4}-q^{3}}\right)^{1 / 3}}\right]
$$

where the quantity $q$ is

$$
q=\left(\frac{16}{27}\right)^{1 / 3} \cdot \frac{U-2}{(\varepsilon)^{2 / 3}} .
$$

The value of $s$ that corresponds to $U=2$ is

$$
s_{\varepsilon}(2)=\left(\frac{\varepsilon}{4}\right)^{1 / 3} .
$$


As we pointed out earlier in the discussion, (5.6) is the relation for the stable solution. For the unstable solution, we have a similar relation

$$
U_{\varepsilon}(r)=\frac{r}{r-\frac{\varepsilon}{4}} \cdot U\left(r-\frac{\varepsilon}{4}\right)
$$

where $U_{\varepsilon}(r)$ corresponds to the unstable solution in the presence of shear flow and function $U(r)$ is for the nematic solution with no shear flow. Below we show that the unstable branch $U_{\varepsilon}(r)$ has a minimum. When $r$ is of the order of $O\left(\varepsilon^{1 / 3}\right)$, expanding (5.14) and neglecting $O\left(\varepsilon^{4 / 3}\right)$ terms yields

$$
U_{\varepsilon}(r)-2=\frac{\varepsilon}{2 r}+\frac{1}{4} r^{2}
$$

In this case, the two terms on the right hand side of (5.15) are of the same order and $U_{\varepsilon}$ is of the order of $2+O\left(\varepsilon^{2 / 3}\right)$. In (5.15), minimizing $U_{\varepsilon}$ with respect to $r$, we obtain that $U_{\varepsilon}$ attains the minimum at $r=\varepsilon^{1 / 3}$. The minimum value of $U_{\varepsilon}$ (the unstable branch) is

$$
\min U_{\varepsilon}=2+\frac{3}{4} \varepsilon^{2 / 3}
$$

For $r<<O\left(\varepsilon^{1 / 3}\right)$, the first term on the right-hand side of $(5.15)$ is the dominant term while for $r>>O\left(\varepsilon^{1 / 3}\right)$, the second term is the dominant term. For both of these two caess, we have $U_{\varepsilon}-2>>O\left(\varepsilon^{2 / 3}\right)$. Therefore, the minimum of $U_{\varepsilon}$ attained at $r=\varepsilon^{1 / 3}$ is the global minimum of $U_{\varepsilon}$. The scaling relation (5.16) is consistent with the one obtained in [23] for the Doi closure. Phase diagrams of the stable branch, the unstable branch and the travelling wave branch (unsteady, periodic in time) for $\varepsilon=0.1$ are shown in Figure 5.2.

\section{Conclusion}

In this paper we have studied analytically the 2D Smoluchowski equation under an imposed weak shear. Our main results can be summarized as follows.

- In the presence of an imposed weak shear there exists a critical value $\left(U_{0}=2.41144646\right)$ for the normalized polymer concentration $U$. Steady state solution exists only for $U<U_{0}$.

- The multi-scale asymptotic analysis describes the slow time evolution driven by the weak shear. It is found that the order parameter of the orientational distribution of rodlike molecules is time-invariant while the director changes with time. It is further shown that when $U<U_{0}$, the director of the orientational distribution converges to a stable steady state position; when $U>U_{0}$, the angular velocity of the director is position-dependent and is always positive. As a result, the orientational distribution is temporally periodic and can never reach a steady state for $U>U_{0}$.

- Finally, it is revealed that the phase relation under weak shear can be obtained from the phase relation with no shear by a simple algebraic transformation.

\section{Appendix A. Proof of Theorem 3.1.}

Below we prove the 4 items in Theorem 1 one by one.

1) It is straightforward to verify that $g(0)=1$ and $g(r)>0$ from (3.13). 

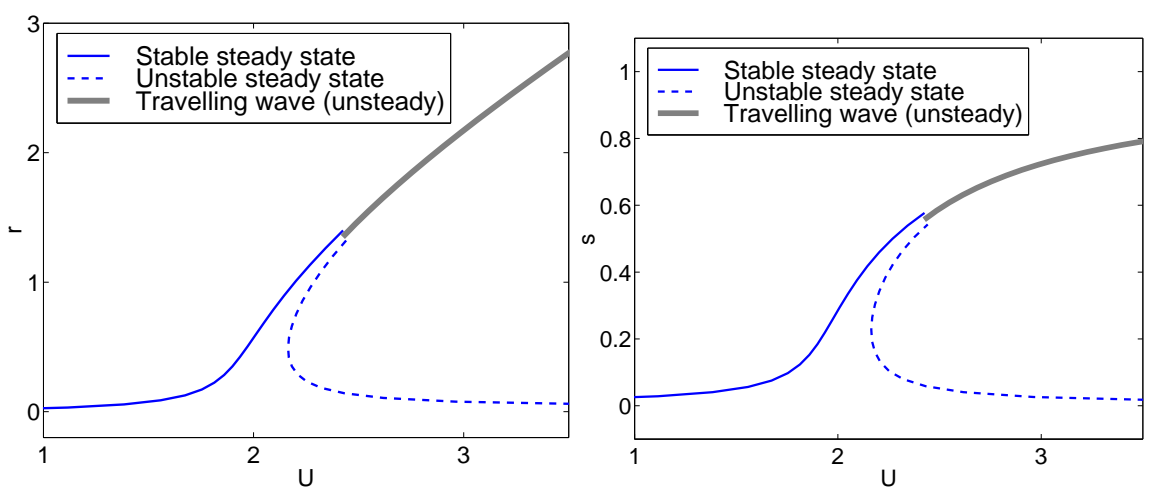

FIG. 5.2. Phase diagrams of the stable steady state, unstable steady state and the traveling wave (unsteady) for the $2 D$ nematic polymers with $\varepsilon=0.1$. Here $\varepsilon$ is the Peclet number. The left panel shows $r$ as a function of the normalized polymer concentration $U$ where $r$ is defined as $r \equiv U\langle\cos 2 \theta\rangle$. The right panel shows the order parameter $s$ as a function of $U$ where the order parameter is defined as $s \equiv\langle\cos 2 \theta\rangle$. Unstable steady state does not exist for $U<2+\frac{3}{4} \varepsilon^{2 / 3}$. For $U>2+\frac{3}{4} \varepsilon^{2 / 3}$, unstable steady states appear. A unstable steady state, when perturbed, will either converge to a stable steady state or converge to a travelling wave (unsteady, periodic in time). The lower half of the unstable steady branch extends to $U=+\infty$, which corresponds to the unstable isotropic state in the absence of shear flow. The upper half of the un stable steady branch stops at $U_{0}=2.41144646$. The stable steady state branch also stops at $U_{0}=2.41144646$. For $U>U_{0}=2.41144646$, there is no steady nematic state (stable or unstable). Instead, there is a travelling wave nematic state (unsteady, periodic in time).

2) Differentiating (3.13), we have

$$
\begin{aligned}
\frac{d}{d r} g(r) & \left.=2\left(\frac{1}{2 \pi} \int_{0}^{2 \pi} \exp (r \cos 2 \theta) d \theta\right)\left(\frac{1}{2 \pi} \int_{0}^{2 \pi} \cos 2 \theta \exp (r \cos 2 \theta)\right) d \theta\right) \\
& =2\left(\frac{1}{2 \pi} \int_{0}^{2 \pi} \exp (r \cos 2 \theta) d \theta\right)^{2} \cdot \frac{\int_{0}^{2 \pi} \cos 2 \theta \exp (r \cos 2 \theta) d \theta}{\left.\int_{0}^{2 \pi} \exp (r \cos 2 \theta)\right) d \theta} \\
& =2 g(r)\langle\cos 2 \theta\rangle .
\end{aligned}
$$

Thus, to show that $g^{\prime}(r)>0$ for $r>0$, we only need to show $\langle\cos 2 \theta\rangle>0$ for $r>0$. Using the probability density given in (2.6), it is straightforward to verify that $\langle\cos 2 \theta\rangle_{r=0}=$ 0 . Differentiating $\langle\cos 2 \theta\rangle$ with respect to $r$, we get

$$
\frac{d}{d r}\langle\cos 2 \theta\rangle=\langle\cos 2 \theta(\cos 2 \theta-\langle\cos 2 \theta\rangle)\rangle=\operatorname{var}(\cos 2 \theta)>0,
$$

where $\operatorname{var}(\cos 2 \theta)$ denotes the variance of random variable $\cos 2 \theta$. It follows that $\langle\cos 2 \theta\rangle$ is an increasing function. In particular, we have $\langle\cos 2 \theta\rangle>0$ for $r>0$.

3) Using equation (2.7), we write function $h(r)$ in the form

$$
h(r)=\frac{1}{\langle\cos 2 \theta\rangle}[1-g(r)(1-\langle\cos 2 \theta\rangle)] .
$$

Using the probability density given in (2.6), it is straightforward to verify that 
$\langle\cos 2 \theta\rangle_{r=0}=0,\left\langle\cos ^{2} 2 \theta\right\rangle_{r=0}=\frac{1}{2}$ and $\left\langle\cos ^{3} 2 \theta\right\rangle_{r=0}=0$. Furthermore, we have

$$
\begin{aligned}
& \left.\frac{d}{d r}\langle\cos 2 \theta\rangle\right|_{r=0}=\left.\left(\left\langle\cos ^{2} 2 \theta\right\rangle-\langle\cos 2 \theta\rangle^{2}\right)\right|_{r=0}=\frac{1}{2}>0, \\
& \left.\frac{d^{2}}{d r^{2}}\langle\cos 2 \theta\rangle\right|_{r=0}=\left.\left\langle\cos ^{2} 2 \theta(\cos 2 \theta-\langle\cos 2 \theta\rangle)\right\rangle\right|_{r=0} \\
& \quad-\left.\left.2\langle\cos 2 \theta\rangle\right|_{r=0} \cdot\langle\cos 2 \theta(\cos 2 \theta-\langle\cos 2 \theta\rangle)\rangle\right|_{r=0}=0, \\
& \left.\frac{d}{d r} g(r)\right|_{r=0}=\left.2 g(r)\langle\cos 2 \theta\rangle\right|_{r=0}=0, \\
& \left.\frac{d^{2}}{d r^{2}} g(r)\right|_{r=0}=\left.\left[2 \frac{d}{d r} g(r)\langle\cos 2 \theta\rangle+2 g(r) \frac{d}{d r}\langle\cos 2 \theta\rangle\right]\right|_{r=0}=1 .
\end{aligned}
$$

Using these results to expand $\langle\cos 2 \theta\rangle$ and $g(r)$ around $r=0$, we have

$$
\begin{aligned}
\langle\cos 2 \theta\rangle & =\frac{1}{2} r+O\left(r^{3}\right) \\
g(r) & =1+\frac{r^{2}}{2}+O\left(r^{3}\right) .
\end{aligned}
$$

Substituting these expansions into (A.3), we obtain

$$
h(r)=1-r+O\left(r^{2}\right) .
$$

Thus, we have $h^{\prime}(0)=-1<0$.

4) In order to find out the asymptotic behavior of $h(r)$ as $r \rightarrow+\infty$, we use Watson's lemma [2]. First, we make a change of variable:

$$
q=-\cos 2 \theta+1 .
$$

As $r \rightarrow+\infty$, the dominant contribution from integral $\int_{0}^{2 \pi} \exp (r \cos 2 \theta) d \theta$ comes from the neighborhood near $\cos 2 \theta=1$ (that is, the neighborhood near $q=0$ ). Differentiating (A.6) and expanding for small $q$, we have

$$
2 d \theta=\frac{1}{\sqrt{2 q}} \frac{1}{\sqrt{1-\frac{q}{2}}} d q=\frac{1}{\sqrt{2 q}}\left(1+\frac{1}{4} q+\cdots\right) d q .
$$

Substituting into integral $\int_{0}^{2 \pi} \exp (r \cos 2 \theta) d \theta$ yields

$$
\begin{aligned}
& \int_{0}^{2 \pi} \exp (r \cos 2 \theta) d \theta=4 \int_{0}^{\pi / 2} \exp (r \cos 2 \theta) d \theta \\
= & 2 \exp (r) \int_{0}^{2} \exp (-r q) \frac{1}{\sqrt{2 q}}\left(1+\frac{1}{4} q+\cdots\right) d q \\
= & \sqrt{2} \exp (r) \int_{0}^{2} \exp (-r q)\left(q^{-\frac{1}{2}}+\frac{1}{4} q^{\frac{1}{2}}+\cdots\right) d q \\
= & \sqrt{2} \exp (r)\left[\frac{\Gamma\left(\frac{1}{2}\right)}{r^{\frac{1}{2}}}+\frac{1}{4} \frac{\Gamma\left(\frac{3}{2}\right)}{r^{\frac{3}{2}}}+\cdots\right] \\
= & \frac{\sqrt{2 \pi}}{\sqrt{r}} \exp (r)\left(1+\frac{1}{8 r}+\cdots\right) \quad \text { as } r \rightarrow+\infty .
\end{aligned}
$$


Similarly, we have

$$
\begin{aligned}
& \int_{0}^{2 \pi} \cos 2 \theta \exp (r \cos 2 \theta) d \theta=4 \int_{0}^{\pi / 2} \cos 2 \theta \exp (r \cos 2 \theta) d \theta \\
= & 2 \exp (r) \int_{0}^{2} \exp (-r q)(1-q) \frac{1}{\sqrt{2 q}}\left(1+\frac{1}{4} q+\cdots\right) d q \\
= & \sqrt{2} \exp (r) \int_{0}^{2} \exp (-r q)\left(q^{-\frac{1}{2}}-\frac{3}{4} q^{\frac{1}{2}}+\cdots\right) d q \\
= & \sqrt{2} \exp (r)\left[\frac{\Gamma\left(\frac{1}{2}\right)}{r^{\frac{1}{2}}}-\frac{3}{4} \frac{\Gamma\left(\frac{3}{2}\right)}{r^{\frac{3}{2}}}+\cdots\right] \\
= & \frac{\sqrt{2 \pi}}{\sqrt{r}} \exp (r)\left(1-\frac{3}{8 r}+\cdots\right) \quad \text { as } r \rightarrow+\infty .
\end{aligned}
$$

The above two expansions lead to

$$
\begin{array}{rlr}
\langle\cos 2 \theta\rangle & =\frac{\int_{0}^{2 \pi} \cos 2 \theta \exp (r \cos 2 \theta) d \theta}{\int_{0}^{2 \pi} \exp (r \cos 2 \theta) d \theta} \\
& =\frac{1-\frac{3}{8 r}+\cdots}{1+\frac{1}{8 r}+\cdots}=1-\frac{1}{2 r}+\cdots \quad \text { as } r \rightarrow+\infty
\end{array}
$$

and

$$
\begin{aligned}
g(r) & =\left(\frac{1}{2 \pi} \int_{0}^{2 \pi} \exp (r \cos 2 \theta) d \theta\right)^{2}=\left[\frac{1}{\sqrt{2 \pi r}} \exp (r)\left(1+\frac{1}{8 r}+\cdots\right)\right]^{2} \\
& =\frac{1}{2 \pi r} \exp (2 r)\left(1+\frac{1}{4 r}+\cdots\right) \quad \text { as } r \rightarrow+\infty
\end{aligned}
$$

Substituting the expansions for $\langle\cos 2 \theta\rangle$ and $g(r)$ into $h(r)$, we arrive at

$$
\begin{aligned}
h(r) & =\frac{1}{\langle\cos 2 \theta\rangle}[1-g(r)(1-\langle\cos 2 \theta\rangle)] \\
& =-\frac{1}{4 \pi r^{2}} \exp (2 r)+\cdots \quad \text { as } r \rightarrow+\infty .
\end{aligned}
$$

This completes the proof of Theorem 3.1.

Acknowledgements. The authors thank Professors Peter Constantin and Gregory Forest for helpful discussions. The authors thank the anonymous referees for their suggestions in improving this manuscript. This work was partially supported by the Naval Postgraduate School Research Initiation Program, the Air Force Office of Scientific Research and the National Science Foundation.

\section{REFERENCES}

[1] B. Bird, R. C. Armstrong and O. Hassager, Dynamics of Polymeric Liquids, Wiley, 1, 1987.

[2] A. W. Bush, Perturbation Methods for Engineers and Scientists, CRC Press, 1992.

[3] E. P. Choate and M. G. Forest, A classical problem revisited: rheology of nematic polymer monodomains in small amplitude oscillatory shear, Rheol. Acta., 46(1), 83-94, 2006. 
[4] F. Cocchini, C. Aratari and G. Marrucci, Tumbling of rod-like polymers in the liquid-crystalline phase under shear flow, Macromolecules, 23, 4076-4082, 1990.

[5] P. Constantin, I. Kevrekidis and E. S. Titi, Asymptotic states of a Smoluchowski equation, Arch. Rat. Mech. Anal., 174, 365-384, 2004.

[6] P. Constantin, I. Kevrekidis and E. S. Titi, Remarks on a Smoluchowski equation, Discrete and Continuous Dynamical Systems, 11, 101-112, 2004.

[7] P. Constantin and J. Vukadinovic, Note on the number of steady states for a 2D Smoluchowski equation, Nonlinearity, 18, 441-443, 2005.

[8] P. Constantin, Nonlinear Fokker-Planck Navier-Stokes systems, Commun. Math. Sci., 3(4), 531-544, 2005.

[9] M. Doi and S. F. Edwards, The Theory of Polymer Dynamics, Oxford University Press, 1986.

[10] Weinan E, Tiejun Li and Pingwen Zhang, Well-posedness for the dumbbell model of polymeric fluids, Comm. Math. Phys., 248, 409-427, 2004.

[11] I. Fatkullin and V. Slastikov, A note on the Onsager model of nematic phase transitions, Commun. Math. Sci., 3, 21-26, 2005.

[12] I. Fatkullin and V. Slastikov, Critical points of the Onsager functional on a sphere, Nonlinearity, 18, 2564-2580, 2005.

[13] M. G. Forest, R. Zhou and Q. Wang, Symmetries of the Doi kinetic theory for nematic polymers of arbitrary aspect ratio: at rest and in linear flows, Phys. Rev. E 66, 031712, 2002.

[14] M. G. Forest, Q. Wang and R. Zhou, The flow-phase diagram of Doi-Hess theory for sheared nematic polymers II: finite shear rates, Rheol. Acta, 44(1), 80-93, 2004.

[15] M. G. Forest, R. Zhou and Q. Wang, The weak shear phase diagram for nematic polymers, Rheol. Acta, 43(1), 17-37, 2004

[16] M. G. Forest, R. Zhou and Q. Wang, Chaotic boundaries of nematic polymers in mixed shear and extensional flows, Physical Review Letters, 93(8), 088301-088305, 2004

[17] M. G. Forest, R. Zhou and Q. Wang, Kinetic structure simulations of nematic polymers in plane Couette cells, I: The algorithm and benchmarks, SIAM MMS, 3(4), 853-870, 2005.

[18] M. G. Forest, R. Zhou and Q. Wang, Scaling behavior of kinetic orientational distributions for dilute nematic polymers in weak shear, J. Non-Newtonian Fluid Mech., 116, 183-204, 2004.

[19] M. G. Forest and Q. Wang, Monodomain response of finite-aspect-ratio macromolecules in shear and related linear flows, Rheol. Acta, 42, 20-46, 2003.

[20] S. Hess and M. Kroger, Regular and chaotic orientational and rheological behaviour of liquid crystals, J. Phys.: Condens. Matter, 16, S3835-S3859, 2004.

[21] E. J. Hinch, Perturbation Methods, Cambridge University Press, 1991.

[22] R. G. Larson, Arrested tumbling in shearing flows of liquid crystal polymers, Macromolecules 23, 3983-3992, 1990.

[23] J. H. Lee, M. G. Forest and R. Zhou, Alignment and rheo-oscillator criteria for sheared nematic films in the monolayer limit, Discrete and Continuous Dynamical Systems - Series B, 6, 339-356, 2006.

[24] H. Liu, H. Zhang and P. Zhang, Axial symmetry and classification of stationary solutions of Doi-Onsager equation on the sphere with Maier-Saupe potential, Commun. Math. Sci., 3, 201-218, 2005.

[25] C. Luo, H. Zhang and P. Zhang, The structure of equilibrium solution of $1 D$ Smoluchowski equation, Nonlinearity, 18, 379-389, 2005.

[26] G. Marrucci and P. L. Maffettone, Description of the liquid-crystalline phase of rodlike polymers at high shear rates, Macromolecules, 22, 4446-4451, 1989.

[27] G. Marrucci and P. L. Maffettone, Nematic phase of rodlike polymers. I. Prediction of transient behavior at high shear rates, J. Rheol. 34, 1217-1230, 1990.

[28] E. Vicente Alonso, A. Wheeler and T. Sluckin, Nonlinear dynamics of a nematic liquid crystal in the presence of a shear flow, Proc. R. Soc. Lond. A, 459, 195-220, 2003.

[29] Q. Wang, S. Sircar and H. Zhou, Solutions of the Smoluchowski equation for nematic polymers under imposed fields, Commun. Math. Sci., 3(4), 605-620, 2005.

[30] A. Zarnescu, The stationary 2D Smoluchowski equation in strong homogeneous flow, Nonlinearity, 19, 1619-1628, 2006.

[31] A. Zarnescu, Patterns in a Smoluchowski equation, preprint.

[32] H. Zhou, H. Wang, M. G. Forest and Q. Wang, A new proof on axisymmetric equilibria of a three-dimensional Smoluchowski equation, Nonlinearity, 18, 2815-2825, 2005. 